\title{
High-Energy Electron Spikes at High Latitudes
}

\author{
J. W. Brown ANd E. C. Stone ${ }^{1}$ \\ California Institute of Technology, Pasadena, California 91109
}

\begin{abstract}
Over 750 spikes of precipitating electrons with $E \geq 425 \mathrm{kev}$ were observed aboard the low-altitude polar orbiter Ogo 4 between July 30, 1967, and December 31, 1967. The spikes may be divided into three distinct populations depending on whether they occur at latitudes below, at, or above the local limit of trapping. These spikes are designated type 1, 2, and 3, respectively. Type 3 spikes occur in a narrow latitude band about $3^{\circ}$ wide, centered at invariant latitude $\Lambda \approx 78^{\circ}$ at 1000 MLT (magnetic local time) and $68^{\circ}$ at 2000 MLT. Their relative frequency of occurrence, intensity, and hardness do not depend significantly on magnetic local time. Type 3 spikes appear to be associated with spikes observed near the magnetopause and the neutral sheet. Type 2 spikes also occur in a latitude band about $3^{\circ}$ wide, centered at about $71^{\circ}$ at $1000 \mathrm{MLT}$ and $67^{\circ}$ at $2200 \mathrm{MLT}$. Their frequency of occurrence is highly dependent on magnetic local time, a large maximum occurring near 2300 and very few events occurring between 0600 and 1200 MLT. Type 2 spikes appear to be related to island fluxes in the neutral sheet, although they occur on closed field lines and may persist for many hours. Type 1 spikes occur in a wider band of latitudes, from about $62^{\circ}$ to $68^{\circ}$ near midnight and $66^{\circ}$ to $68^{\circ}$ near noon. The local-time dependence of their frequency of occurrence is similar to that of type 2 spikes but less pronounced. Although they are observed on closed field lines, type 1 spikes do not persist for periods longer than about 1 hour, and we conclude that they are produced by strong pitch-angle scattering from the stably trapped population. The average spectral index (assuming a power-law spectrum) is $\mathbf{3}$ to $\mathbf{5}$, and the median flux (>425 kev) is about $150 \mathrm{el} \mathrm{cm}^{-2} \mathrm{sec}^{-1} \mathrm{ster}^{-1}$, although type 2 events near midnight tend to be larger and harder. All types tend to be more intense and to occur at lower latitudes when $K p$ is large.
\end{abstract}

We will discuss phenomena known as 'electron spikes' or 'particle spikes.' These spikes are defined as brief, rapid increases in counting rate as observed by a particle detector, in this instance aboard a low-altitude polarorbiting spacecraft. Numerous experimenters have observed spikes of electrons at high latitudes, ranging in energy from 0.7 [Hoffman and Evans, 1968; Hoffman, 1969] to above $500 \mathrm{kev}$ [Anderson et al., 1968; McCoy, 1969]. In addition, electron 'islands' having similar characteristics have been reported in the geomagnetic tail [Montgomery et al., 1965; Anderson, 1965; Murayama and Simpson, 1968; Retzler and Simpson, 1969], in the vicinity of the bow shock [Frank and Van Allen, 1964; Anderson et al., 1965], and near the equatorial plane at moderate distances (5-10 $\left.R_{E}\right)$ [Frank, 1965; Rosen, 1965; Arnoldy and Chan, 1969]. It is difficult to make definite connections be-

1 Alfred P. Sloan Research Fellow.

Copyright (C) 1972 by the American Geophysical Union. tween these various events because of the small probability of having several spacecraft properly positioned and aligned at the time an event occurs. One such identification has apparently been made by Hones et al. [1971], who observed a spike simultaneously at $1,6.6$, and $18 R_{B}$.

Spike observations at low altitudes by polarorbiting spacecraft offer the advantage of frequent sampling of field lines where spikes are usually seen. This advantage makes possible statistical studies of the distribution and behavior of these events, which are necessary to determine the effect of spikes on the energy budget and particle populations within the magnetosphere and their relationships to other processes. The purpose of this paper is to present such a statistical study in which the effects of relevant physical parameters are represented with sufficient accuracy to be a useful basis for further theoretical calculations.

It should be emphasized that the present results represent events with electron energies $E$. $\geq 425 \mathrm{kev}$. There seems to be very little correlation between spikes in this energy range 
and those observed by Hoffman and Evans [1968] in the energy range $0.7 \leq E_{0} \leq 24 \mathrm{kev}$. Likewise, the phenomena observed here differ significantly from those reported by McDiarmid and Burrows [1965] for $E_{0} \geq 40 \mathrm{kev}$. However, at least part of our observations may be related to those of Fritz and Gurnett [1965] for $E_{e} \geq 10 \mathrm{kev}$. We will examine the relationships among these observations, especially with respect to various possible source mechanisms.

\section{Description OF EXPERIMENT}

The Ogo 4 spacecraft was launched into a low-altitude polar orbit on July $28,1967$. Initial orbit parameters were: apogee $908 \mathrm{~km}$, perigee $412 \mathrm{~km}$, inclination $86^{\circ}$, period $98 \mathrm{~min}$. The spacecraft was oriented so that the University of Chicago/California Institute of Technology vertical particle telescope (experiment D-08) faced radially away from the earth. The experiment also included a horizontal detector that was insensitive to electrons and that will not be discussed here.

A cross section of the vertical telescope is shown in Figure 1. The detector system is described in detail by Evans et al. [1970]. The opening half-angle of $30^{\circ}$, combined with the radial orientation of the symmetry axis, ensures that the telescope is responding primarily to precipitating particles at invariant latitudes $\Lambda \gtrsim 45^{\circ}$. Mirroring particles will be detected at these latitudes only if they scatter from the telescope wall.

Table 1 indicates the response of the telescope to various particle types. The upper cutoff energies in each instance are set by the anticoincidence scintillator $D_{3}$. The rates indicated in Table 1 are monitored, and the particle energy loss in $D_{1}$ is pulse-height analyzed for each event satisfying the $D_{1} D_{3}$ requirement. A flag is set

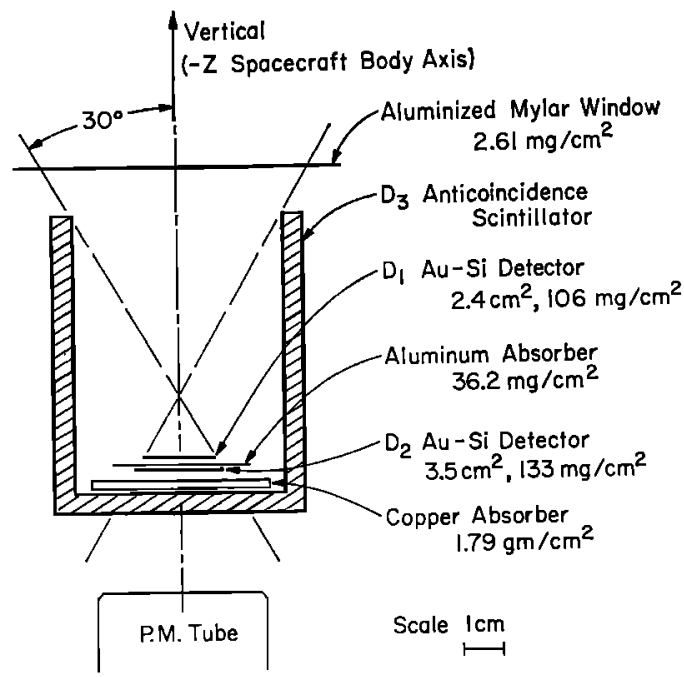

Fig. 1. Ogo 4 vertical particle telescope cross section. Not shown is a magnesium wall that covers the inner side of the scintillator. The $\mathrm{D}_{3}$ anticoincidence scintillator is surrounded by 138 $\mathrm{mg} / \mathrm{cm}^{2}$ of magnesium. $D_{1}$ and $D_{2}$ both have depletion depths of $56 \mathrm{mg} / \mathrm{cm}^{2}$.

for each analyzed event to indicate whether or not $D_{2}$ was triggered in coincidence with $D_{1}$. Because of the electronic thresholds of the detectors (425 kev for $\mathrm{D}_{1}$ and $250 \mathrm{kev}$ for $\mathrm{D}_{2}$ ), electrons that leave enough energy in $D_{1}$ to be detected cannot reach $\mathrm{D}_{2}$, and electrons that leave enough energy in $D_{2}$ to be detected will not have left enough in $D_{1}$. Therefore there is a very low probability that electrons cause any $\mathrm{D}_{1} \mathrm{D}_{2}$ true coincidences, and we know we are observing mostly electrons whenever the $\mathrm{D}_{2} \overline{\mathrm{D}}_{3}$ rate is large as compared with the $\mathrm{D}_{1} \mathrm{D}_{2} \mathrm{D}_{3}$ rate. Pulse-height data have been used in several instances to confirm these conclusions.

The basic data used in the present work are plots of the $\mathrm{D}_{3}$ rate averaged over 1.44-sec intervals and the $\mathrm{D}_{1} \overline{\mathrm{D}}_{3}, \mathrm{D}_{2} \overline{\mathrm{D}}_{3}$, and $\mathrm{D}_{1} \mathrm{D}_{2} \overline{\mathrm{D}}_{3}$

TABLE 1. Detector Response

\begin{tabular}{lccccc}
\hline Detector(s) & $p, \mathrm{Mev}$ & $\alpha, \mathrm{Mev}$ & $e^{*}, \mathrm{Mev}$ & $\begin{array}{c}A \Omega_{p, \alpha}, \\
\mathrm{cm}^{2} \text { ster }\end{array}$ & $\begin{array}{c}A \Omega_{e}(\max .), \\
\mathrm{cm}^{2} \text { ster }\end{array}$ \\
\hline $\mathrm{D}_{1} \overline{\mathrm{D}}_{3}$ & $1.22-39.2$ & $4.88-157$ & $0.45-1.8$ & $1.05-1.18$ & 0.08 \\
$\mathrm{D}_{2} \overline{\mathrm{D}}_{3}$ & $9.32-39.2$ & $37.3-157$ & $0.7-4$ & $1.50-1.56$ & 0.36 \\
$\mathrm{D}_{1} \mathrm{D}_{2} \overline{\mathrm{D}}_{3}$ & $9.32-39.2$ & $37.3-157$ & & $1.05-1.18$ & 0 \\
$\mathrm{D}_{3}$ & $\gtrsim 9.3$ & $\gtrsim 37$ & $\gtrsim 0.5$ & & \\
\hline
\end{tabular}

\footnotetext{
* Electron energies at which $A \Omega_{\mathrm{e}} \gtrsim 0.5 A \Omega_{\mathrm{e}}$ (max.).
} 
rates averaged over approximately 15 -sec intervals. These data and the descriptive text [Evans et al., 1970] will be submitted to the National Space Science Data Center.

\section{Spectrad Response}

A simulation of the detector system was exposed to electrons from a $\beta$ source. Monoenergetic electrons were selected by means of a magnetic spectrometer. The true flux of particles at each energy was measured by using a thick total- $E$ detector. The outputs of the telescope detectors were pulse-height analyzed, and the detection efficiency was determined as a function of discriminator threshold. The detection efficiency for discriminator thresholds of $425 \mathrm{kev}$ for $D_{1}$ and $250 \mathrm{kev}$ for $D_{2}$ multiplied by the geometrical factors for $D_{1} \bar{D}_{3}$ and $D_{2} \bar{D}_{3}$, give effective geometrical factors as functions of energy [Lupton and Stone, 1972]. We determined that approximately $50 \%$ of the $\mathrm{D}_{1} \mathrm{D}_{3}$ response to an isotropic flux is due to electrons scattered from the magnesium wall of the telescope but that only approximately $20 \%$ of the $\mathrm{D}_{2} \overline{\mathrm{D}}_{8}$ response arises from this source.

These functions were approximated by piecewise power-law fits over various energy intervals and were folded with various assumed power-law energy spectra to obtain expected counting rates in each detector as functions of the incident electron spectrum. The same process was carried out for various assumed exponential spectra by using exponential fits to the effective geometrical factors. By using these computations it is possible to obtain a limited amount of spectral information from the ratio $\mathrm{D}_{1} \overline{\mathrm{D}}_{3} / \mathrm{D}_{2} \overline{\mathrm{D}}_{3}$.

Preliminary calculations using the results of Fan et al. [1966] indicated that pile up in $\mathrm{D}_{1}$ might be important for some of the largest spikes encountered. The pulse pile-up effect is important only for electrons in $D_{1}$, where the threshold is set electronically. The $\mathrm{D}_{2}$ threshold is set primarily by the requirement that the particle have sufficient range to penetrate $D_{1}$ and the aluminum absorber and still leave $250 \mathrm{kev}$ in $\mathrm{D}_{2}$. Thus the $\mathrm{D}_{2} \mathrm{D}_{3}$ counting rate cannot be due to the pile up of lower-energy ( $\$ 600 \mathrm{kev}$ ) electrons. To estimate the size of the pile-up effect, we performed Monte Carlo calculations of the response of $D_{1}$ to various input fluxes. The result of the calculation was, as one would expect, that the $\mathrm{D}_{1} \mathrm{D}_{3}$ count rate includes a significant contribution from pile-up effects when that rate is larger than $\sim 100 \mathrm{sec}^{-1}$. Since $D_{2}$ is unaffected by pile up, the $D_{2} \bar{D}_{3}$ count rate (corrected for dead time) can be relied on at all reasonable count rates.

\section{Description and Classification of Spikes}

Particle spikes are generally defined by their appearance as narrow peaks in plots of counting rate versus time (or some other parameter, such as invariant latitude, that varies smoothly with time). It is impossible to determine, when looking at an individual spike, whether the rapid variation is a spatial or a temporal phenomenon. Observed on a low-altitude polar-orbiting spacecraft, spikes generally have 'widths' of a few seconds, corresponding to a few tenths of a degree in latitude. High time-resolution ion chamber data [McCoy, 1969; M. J. George, personal communication, 1970] indicate typical widths of $0.25^{\circ}$ to $2^{\circ}$ in invariant latitude or of about 5 to 40 sec in universal time. Thus our averaging time of $15 \mathrm{sec}$ in the count-rate plots may obscure some of the smaller, narrower spikes. For this reason the $D_{8}$ rate plot (with shorter averaging time) occasionally shows spikes that are not visible in the $D_{1} \bar{D}_{3}$ or $D_{2} \bar{D}_{3}$ rates.

We have observed a few spikes for which higher data rates were available. Because of the increase by a factor of 8 in bit rate, pulse-height data from these events could be examined with reasonable counting statistics by using a 2 -sec time resolution. Four events thus examined had widths (FWHM) of about $6,8,10$, and $40 \mathrm{sec}$, as indicated by the electron channels of the $D_{1}$ pulse-height analyzer. (Owing to the large statistical fluctuations in the electron energy loss process, pulse-height data from this experiment yield only minimal spectral ${ }^{r}$ information for electrons. However, pulse-height information does provide positive identification of electrons.) Figure 2 shows two of these events. Notice that the $D_{a}$ rate does not track exactly with the $D_{1} D_{3}$ rate, probably because $D_{1}$ and $D_{3}$ have different energy and angular response characteristics.

Disagreement between McDiarmid and Burrows [1965] and McCoy [1969] about the $\Lambda$-MLT occurrence of spikes and the observation by $M c C o y$ [1969] that the local-time dependence for spikes occurring above the trapping boundary is different from that for 


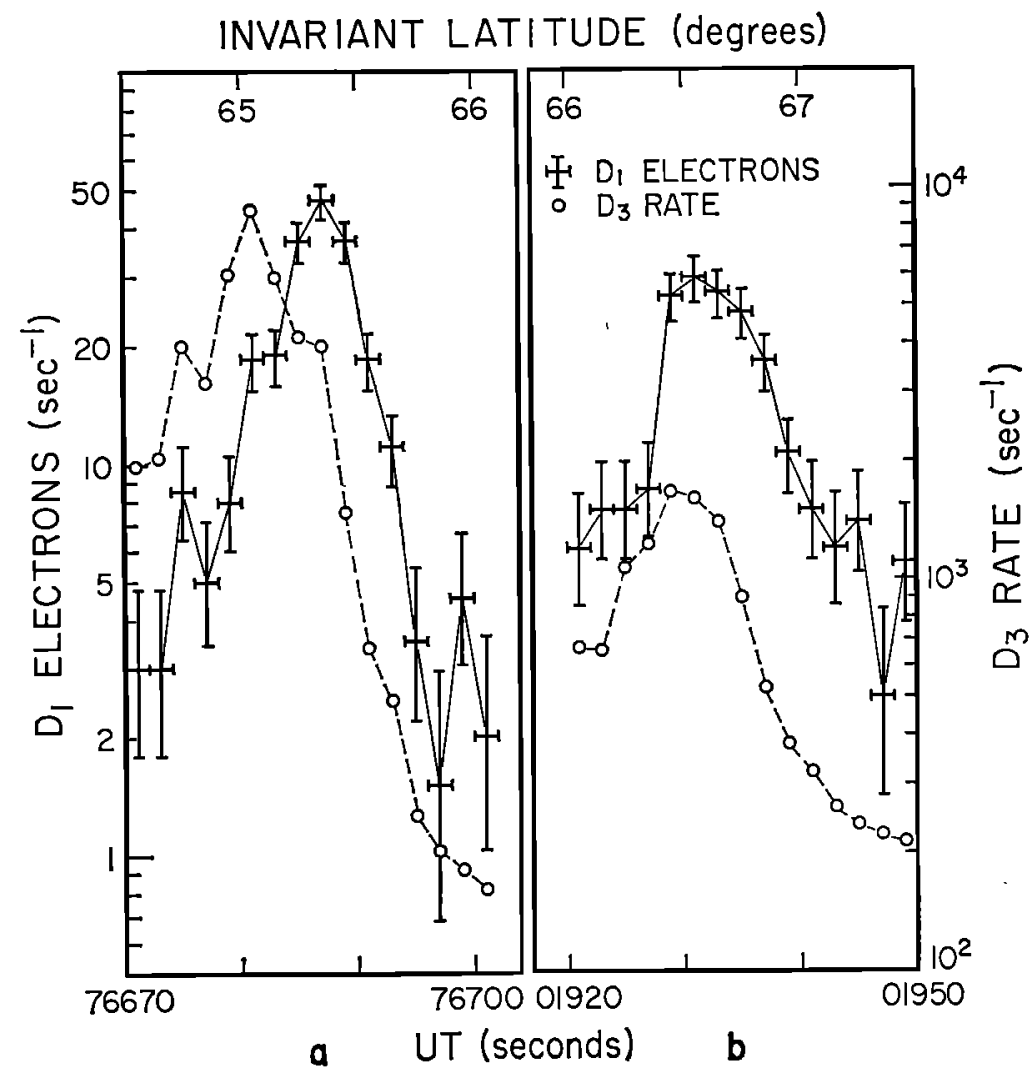

Fig. 2. Real-time data from revolutions 2878 and 2880 , showing large spikes. $D_{1}$ data include only the events that pulse-height analysis shows to be electrons. The events shown occurred at 0120 and 0125 MLT and had widths (FWHM) of 6 and 10 sec. Neither of these events is included in the rest of the data presented. (a) Revolution 2878, February 8, 1968, 0120 MLT. (b) Revolution 2880, February 9, 1968, 0125 MLT.

those below suggest that there may be several distinct populations of spikes and that each should be examined separately. We divided the spikes we observed into three types, according to whether they occur below, at, or above the outer-zone boundary determined for each pass. These types will be referred to as type 1,2 , and 3 , respectively. In addition, a subdivision was made of the type 2 spikes on the basis of their appearance on the rate plots. Some of the spikes show a very sharp boundary on the poleward side, and these spikes are designated as type $2 \mathrm{~A}$. Spikes of this type were observed only at the trapping boundary. Examples of each type are shown in Figure 3. Unless specified otherwise, references to type 2 spikes do not include type $2 \mathrm{~A}$.

The location of a spike relative to the trapping boundary was determined from the $D_{3}$ rate, since this rate had a finer resolution and thus usually showed the most detail. The boundary was defined as the point at which the $D_{3}$ rate reached the polar plateau level if there was no spike obscuring that point. If a spike was overlapping the point where the boundary seemed to be, that spike was designated type 2 . Since this method requires visual inspection and some subjective decision, there is some ambiguity in several instances, and it is possible that a few of the spikes have been assigned to the wrong population. This ambiguity has not caused any obvious difficulties, but it should be remembered when examining the results, especially near local midnight, where there is considerable overlap.

McCoy [1969] has observed that, although 

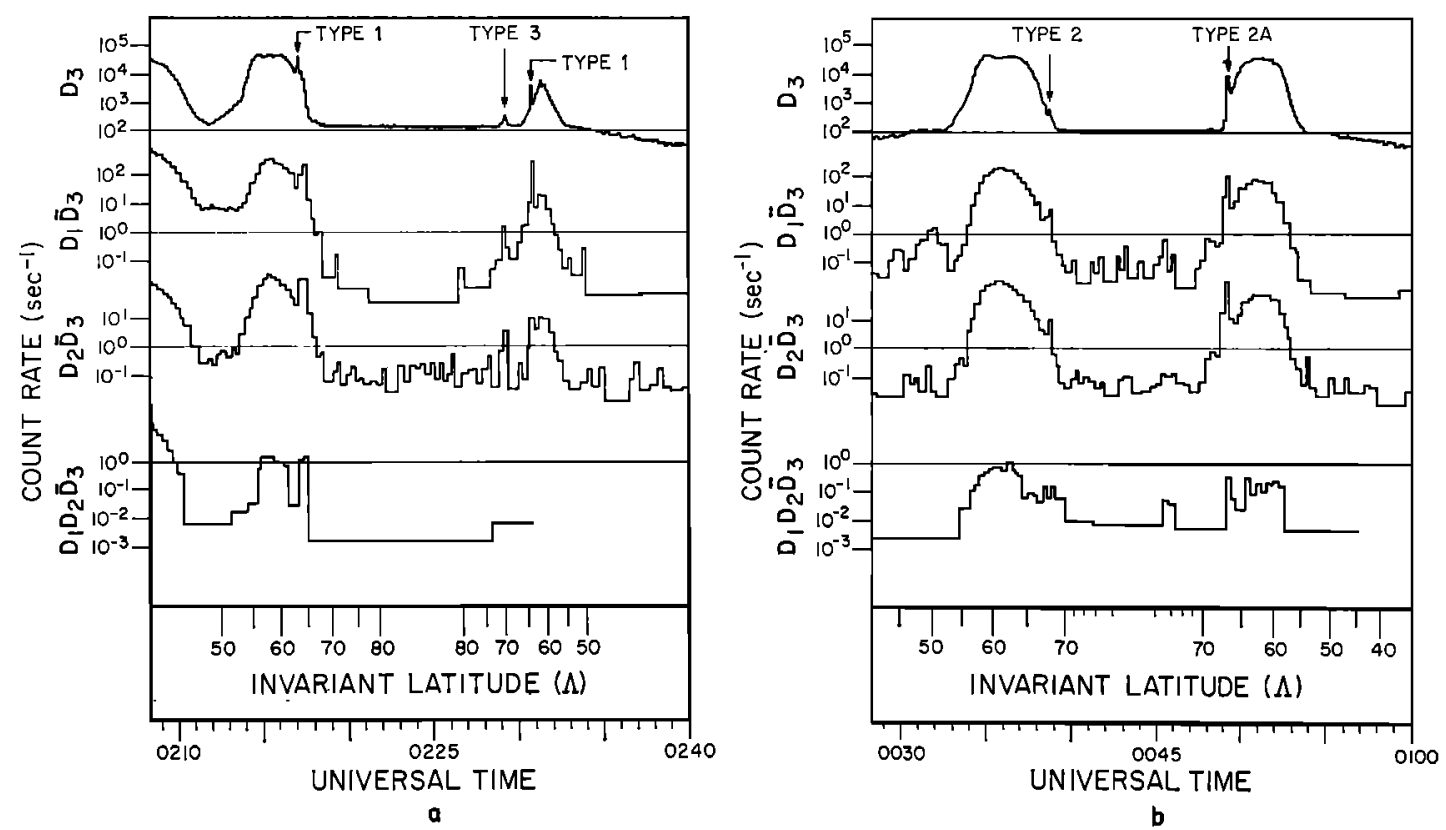

Fig. 3. Examples of Ogo 4 data plots from south polar passes showing spikes of each type. Notice that the $D_{1} D_{2} \bar{D}_{3}$ rate is much smaller than the $D_{2} \overline{\mathrm{D}}_{3}$ rate for each spike, the large disparity indicating electrons. (a) Revolution 934, day 273, September 30, 1967. (b) Revolution 1979, day 344, December 10, 1967.

most spikes do not recur on successive passes, in some instances a spike may be observed twice at a pair of nearly conjugate points, and in a few rare instances the 'same' spike may be present and detectable for several hours. We confirm this observation. In fact, on two occasions we observed what appear to be related series of events lasting over 11 hours, and we observed several groups of shorter duration. All of the larger groups occurred between 2200 and 0100 MLT and within $1^{\circ}$ of the outer-zone boundary. Some of the smaller groups, having observable lifetimes of about 2 hours, occurred nearer the dawn-dusk meridian. The development of one of the large groups is illustrated in Figure 4. This behavior would seem to indicate that the time scales associated with spikes are of the order of minutes to several hours and that the rapid rise and fall characteristic of a spike observed during a given pass represents a purely spatial effect. Parameters associated with these observations are listed in Table 2. The $\Lambda$ values listed in this table have a standard deviation of nearly $1^{\circ}$ which indicates approximately the limit of ac- curacy of the values used throughout this paper. Errors of this magnitude arise from the field model [Jensen and Cain, 1962] used to calculate $L$ and the 6 - to 10 -sec resolution (about $0.3^{\circ}-0.6^{\circ}$ ) associated with reading spike times from data plots.

\section{Data}

We examined all the data available from revolution 24 (July 30, 1967) to revolution 2293 (December 31, 1967). During this period the experiment was turned on about $50 \%$ of the time. Owing to the location of ground stations, we lack (tape-recorded) play-back data for many passes at latitudes where spikes usually occur. We are thus left with approximately 2400 passes (maximum of four passes per orbit) through the spike region, i.e., invariant latitudes $60^{\circ} \leq \Lambda \leq 78^{\circ}$, for which we have useful data. All local times have been sampled without any obvious bias, and so it is tempting to assume that the data represent uniform coverage of all local times. We will make this assumption for the moment, but a detailed 


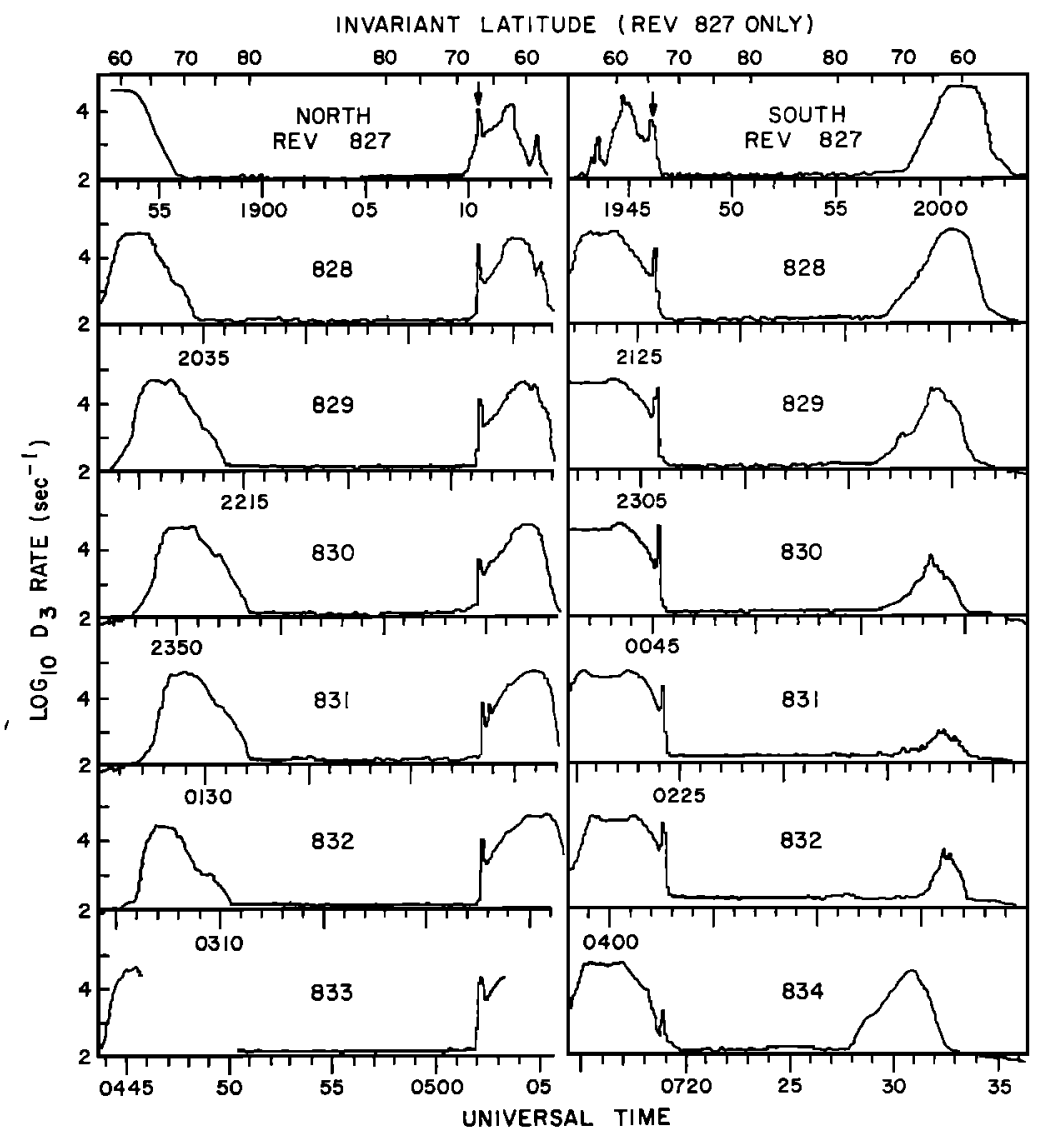

Fig. 4. Development of a long-lasting event of September 22 to 23, 1967. The arrows indicate the position of the spike. Data for revolutions 826 and 835 show no spike, so we assume that these data represent the complete history of the event. The sharp cutoff on the poleward side from revolution 828 to revolution 833 is characteristic of type $2 \mathrm{~A}$ spikes. Notice the more gradual blending into the polar rate in revolutions 827 and 834 . Invariant latitude is indicated for revolution 827 only. See Table 2 for details of each spike.

discussion of the coverage problem will be presented in a later section.

The data to be presented here represent 774 spike observations, of which about 50 to 100 probably are multiple observations of a smaller number of long-lasting events. Figure 5 shows all of the spikes plotted in coordinates of invariant latitude $(\Lambda)$ versus magnetic local time (MLT). (Invariant latitude is defined as cos $\Lambda=(1 / L)^{1 / 2}$, with $L$ calculated from the Jensen and Cain 1960 [Jensen and Cain, 1962] field. See Fritz and Gurnett [1965] for a definition of magnetic local time, but note that the vector products in the denominator of their equation should be cross products.) Notice the significant local-time asymmetry: there are comparatively few events between 0600 and 1200 , whereas there is a large maximum between 2200 and 0400 . Notice also that there are very few events that seem to be on open field lines and that the events cluster about the trapping limit.

Figure 6 shows the same data mapped along field lines into the equatorial plane. The mapping used is that of Fairfield [1968]. Included for later discussion are regions where other investigators have observed spikes.

The first step in analyzing the data was to take a detailed look at the $\Lambda$-MLT dependence for each population. In doing so it was found that, although some very interesting patterns emerged, there was still a large amount 
TABLE 2. Parameters Associated with the Persistent Spike Shown in Figure 4

\begin{tabular}{lccrrr}
\hline Rev. & \multicolumn{1}{c}{$\mathrm{UT}^{*}$} & $\Lambda$ & $\mathrm{MLT}$ & $\mathrm{D}_{\mathbf{1}} \overline{\mathrm{D}}_{3} \dagger$ & $\mathrm{D}_{2} \overline{\mathrm{D}}_{3} \dagger$ \\
\hline $827 \mathrm{~N}$ & 1910.3 & 67.0 & 1.1 & 67 & 107 \\
$827 \mathrm{~S}$ & 1946.0 & 65.2 & 0.8 & 97 & 98 \\
$828 \mathrm{~N}$ & 2048.3 & 67.3 & 1.6 & 16 & 250 \\
$828 \mathrm{~S}$ & 2126.0 & 67.2 & 0.2 & 250 & 250 \\
$829 \mathrm{~N}$ & 2226.4 & 67.9 & 2.1 & & \\
$829 \mathrm{~S}$ & 2305.7 & 67.1 & 23.7 & 250 & 320 \\
$830 \mathrm{~N}$ & 0004.8 & 68.3 & 2.3 & & \\
$830 \mathrm{~S}$ & 0045.3 & 67.3 & 23.6 & 490 & 620 \\
$831 \mathrm{~N}$ & 0143.4 & 69.3 & 2.1 & & \\
$831 \mathrm{~S}$ & 0224.0 & 66.7 & 0.0 & 390 & 620 \\
$832 \mathrm{~N}$ & 0323.0 & 68.0 & 1.4 & 24 & 75 \\
$832 \mathrm{~S}$ & 0402.6 & 67.2 & 0.8 & 400 & 500 \\
$833 \mathrm{~N}$ & 0502.2 & 67.8 & 0.7 & & \\
$833 \mathrm{~S}$ & & & & & \\
$834 \mathrm{~N}$ & & & & & 14 \\
$834 \mathrm{~S}$ & 0719.0 & 68.5 & 3.0 & 8 & 14 \\
\hline
\end{tabular}

* Hours, minutes, tenths.

† Counting rates, counts per second.

of scatter in the data. It was thought that some of the variation in latitude at a given local time might be related to geomagnetic activity. To check this assumption, spikes of each type were grouped into local-time bins such that the variation of $\Lambda$ with MLT within each bin was no larger than the scatter in $\Lambda$.

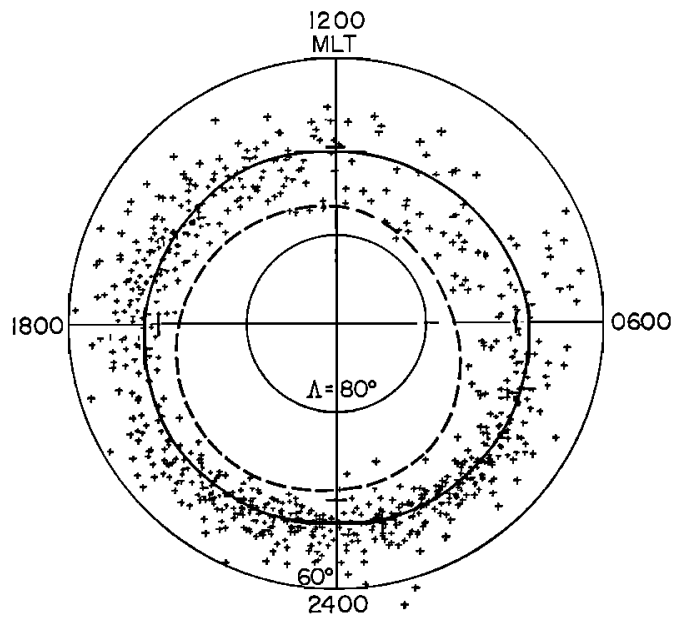

Fig. 5. Latitude-local-time plot of all spike observations. Notice the relative scarcity of events between 0600 and 1200 MLT and the clustering of events near the average trapping boundary. Boundaries are those of McDiarmid and Burrows [1968], determined from 35-kev electron data. Dashed line, limit of closed field lines; solid line, limit of stable trapping.
Plots were then made of $\Lambda$ versus $K p$ for the spikes in each bin, and it became apparent that a significant effect was indeed present. One such plot is shown in Figure 7. Since the data were broken down into so many bins, statistics became poor and justified nothing more than a straight-line fit to the $\Lambda-K p$ dependence in each bin. A linear regression analysis was performed on the data in each bin, and the results are shown in Table 3. By using these results with some smoothing, it is possible to define a new latitude, $\Lambda^{\prime}$, which is the latitude of occurrence of a spike corrected to $K p=0$. This new latitude reduced the scatter at a fixed magnetic local time but only by about $10 \%$ to $30 \%$.

Plots of $\Lambda^{\prime}$ versus magnetic local time are shown in Figure 8. Several observations can be made about these plots. First, it seems reasonable to assume that the strong dependence of $\Lambda^{\prime}$ on MLT for type 3 spikes is due to the distortion of the outer magnetosphere. Second, it can be seen that the high rate of occurrence of spikes near local midnight is due primarily to type 2 spikes, especially those of type $2 \mathrm{~A}$. Notice the pronounced clustering of the type $2 \mathrm{~A}$ spikes near local midnight.

In an attempt to determine whether the remaining scatter in $\Lambda^{\prime}$ could be attributed to any systematic effect, the local-time variations, represented by Fourier-series fits to the data in Figure 8 , were subtracted. The resulting quantity was plotted against universal time and against the angle between the earth's dipole axis and the earth-sun line. No dependence was found in either instance. This lack of dependence leads us to suspect that the remaining scatter is due to (1) inaccuracy in the model of the internal field [Jensen and Cain, 1962] used to generate the original $\Lambda$ values, (2) mislabelling of some of the spikes, (3) errors of a few seconds in reading times from data plots, (4) neglect of the external field in calculating $\Lambda$, or (5) true spatial fluctuations in the process that produces the spikes. Since this remaining scatter is large as compared with the reduction in scatter achieved by extrapolating to $0 K p$, the remainder of the discussion will not include this correction.

The distributions of the intensities and spectral hardnesses of the spikes are of interest for comparison with possible production mechanisms. 


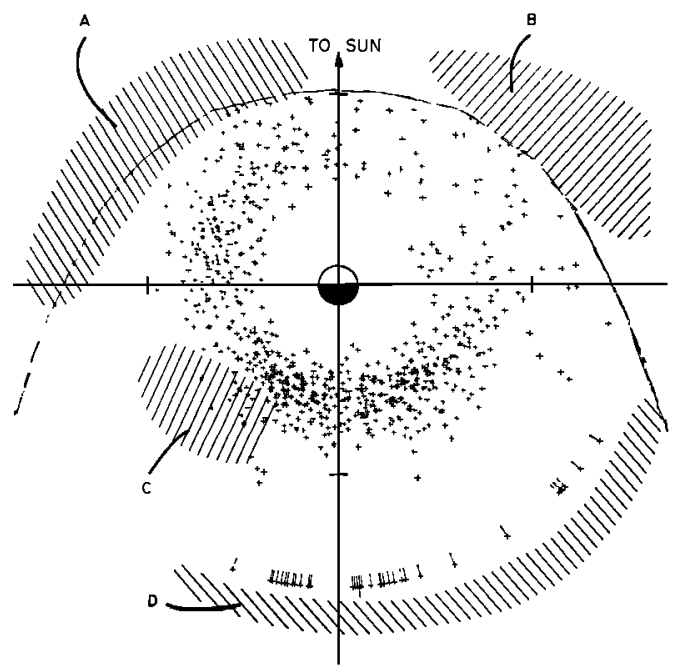

Fig. 6. Data from Figure 5 mapped along field lines into the magnetic equatorial plane using the results of Fairfield [1968]. Points with tick marks attached were outside the range of the mapping and probably should be moved in the direction indicated by the ticks. Regions where other experimenters have observed 'island fluxes' of electrons far from the earth are included for comparison: A, Frank and Van Allen [1964] ; B, Anderson et al. [1965]; C, Rosen [1965]; D, Montgomery et al. [1965]. Tick marks on axis lines are at \pm 10 $R_{E}$. Local-time orientation is the same as in Figure 5.

The peak counting rates $\mathrm{D}_{1} \overline{\mathrm{D}}_{3}$ and $\mathrm{D}_{2} \overline{\mathrm{D}}_{3}$, corrected for background, have been determined for each spike. Owing to the 15-sec averaging, these rates will be underestimates of the true peak values for narrow spikes but will be good estimates for the average flux. We find that the type $2 \mathrm{~A}$ spikes seem to be much harder spectrally than the others. The type 3 spikes may be slightly softer on the average than either the type 1 or the type 2 spikes.

It is not surprising to find that larger events tend to occur during magnetically disturbed periods. There is a slight correlation between the $\mathrm{D}_{1} \overline{\mathrm{D}}_{3}$ rate and $K p$ value, but plots of $\mathrm{D}_{1} \overline{\mathrm{D}}_{3} /$ $\mathrm{D}_{2} \mathrm{D}_{3}$ against $K p$ indicate no significant derendence, except for a slight positive correlation probably due to the pile-up effect in the $\mathrm{D}_{1} \mathrm{D}_{3}$ rate.

Finally, Figure 9 shows the number distribution of spike intensities. The levelling off at small values of $D_{1} \bar{D}_{3}$ is probably due in part to the difficulty of detecting small spikes and should not be considered significant.
Magnetic Local Time and $K p$ Dependence

To obtain the correct dependence of spike occurrence on parameters such as magnetic local time and $K p$, it is necessary to correct the observations for nonuniform sampling. It is common practice, when treating data of this kind from a polar orbiter, to make a cursory check to see that all local times have been sampled more or less uniformly and then to assume that the coverage is indeed uniform and random. This procedure may conceal some peculiarity in the orbit or accidental correlations between orbit parameters and telemetry coverage.

To investigate these possibilities and to correct for them, we employed the following procedure (similar to that used by Fritz and Gurnett [1965]). Data plots were examined to determine times for which usable data were available and processed, regardless of whether or not spikes were present. Then spacecraft attitude-orbit data for these times only and for $\Lambda>55^{\circ}$ were selected for further processing. The orbit sections thus selected were examined in detail, and their distributions in $\Lambda$-MLT-Kp

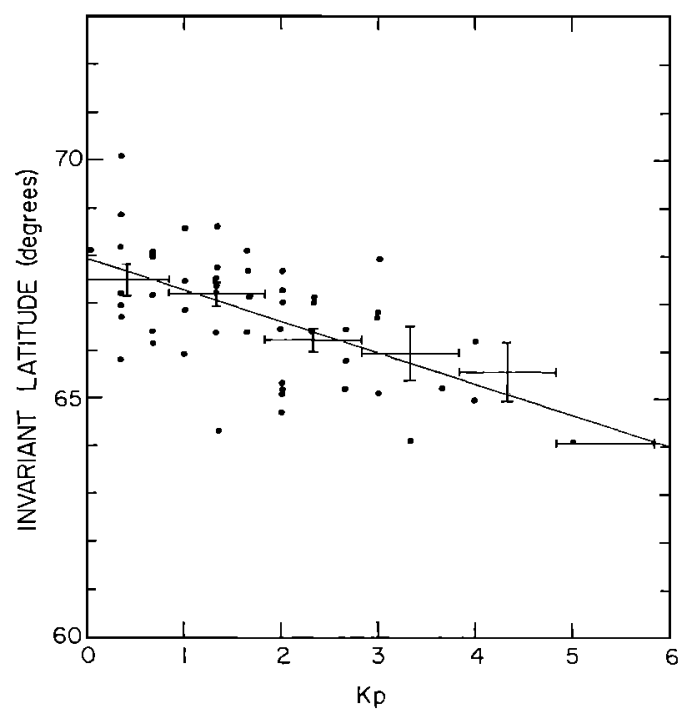

Fig. 7. Sample plot of $\Lambda$ versus $K p$ for a limited range of magnetic local time (0000-0400), showing the tendency for spikes (type 2A) to occur at lower latitudes during magnetically disturbed times. Straight line, a least-squares fit to the points shown; horizontal bars, the points included in the means; vertical bars, the standard deviations of the means. 
TABLE 3. Results of Regression Analysis: Linear Fit of $\Delta$ versus $K p$ in Various Intervals of Magnetic Local Time

\begin{tabular}{|c|c|c|c|c|}
\hline Type & MLT & $\begin{array}{c}\text { Number } \\
\text { of } \\
\text { Spikes }\end{array}$ & $\langle\Lambda\rangle$ & $\begin{array}{c}\text { Slope } \\
(d \Lambda / d K p)\end{array}$ \\
\hline 1 & $0000-0400$ & 44 & 65.0 & $+0.03 \pm 0.26$ \\
\hline 1 & $0400-0900$ & 15 & 64.2 & $+0.09 \pm 0.27$ \\
\hline 1 & $0900-1600$ & 22 & 66.8 & $-0.48 \pm 0.22$ \\
\hline 1 & $1600-2100$ & 40 & 64.3 & $-0.92 \pm 0.27$ \\
\hline 1 & $2100-2400$ & 38 & 65.3 & $-0.97 \pm 0.19$ \\
\hline 2 & $0000-0400$ & 88 & 67.3 & $-0.39 \pm 0.12$ \\
\hline 2 & $0400-0800$ & 31 & 68.6 & $-0.83 \pm 0.18$ \\
\hline 2 & 0800-1500 & 27 & 69.5 & $-0.23 \pm 0.23$ \\
\hline 2 & $1500-1900$ & 59 & 67.8 & $-0.81 \pm 0.17$ \\
\hline 2 & $1900-2400$ & 89 & 66.4 & $-0.61 \pm 0.12$ \\
\hline $2 \mathrm{~A}$ & $0000-0400$ & 54 & 66.8 & $-0.65 \pm 0.14$ \\
\hline $2 \mathrm{~A}$ & 0400-1900 & 13 & 68.4 & $-0.82 \pm 0.42$ \\
\hline $2 \mathrm{~A}$ & $1900-2400$ & 68 & 66.2 & $-0.68 \pm 0.15$ \\
\hline 3 & $0000-0400$ & 30 & 68.1 & $-0.11 \pm 0.29$ \\
\hline 3 & $0400-0700$ & 32 & 70.3 & $-0.07 \pm 0.29$ \\
\hline 3 & $0700-0900$ & 16 & 73.4 & $-0.62 \pm 0.32$ \\
\hline 3 & $0900-1100$ & 12 & 75.9 & $-0.49 \pm 0.37$ \\
\hline 3 & $1100-1300$ & 14 & 74.0 & $-1.06 \pm 0.43$ \\
\hline 3 & $1300-1700$ & 57 & 70.6 & $-0.90 \pm 0.28$ \\
\hline 3 & $1700-2400$ & 25 & 68.3 & $-0.55 \pm 0.33$ \\
\hline
\end{tabular}

space were computed, summarized, and used to normalize the spike occurrences.

Figure 10 shows the distribution in magnetic local time of the occurrences of spikes of various types. The comparison between the raw number of occurrences and the normalized data gives some indication of the importance of the normalization. For instance, it indicates that the apparent local maximum in the occurrence of type 3 spikes near 0500 MLT is an effect of nonuniform coverage. This finding, combined with the possibility of a few misidentified spikes at other local times, indicates that the local-time dependence of type 3 spikes is not significantly different from a uniform distribution in the dawn hemisphere. The peak near $1300 \mathrm{MLT}$ may be related to the position of the neutral point, as will be discussed below. The over-all local-time behavior is similar to that obtained by Fritz and Gurnett [1965] for electrons with $E_{0} \geq$ $10 \mathrm{kev}$. The absolute numbers are not directly comparable, however, owing to the difference in energy thresholds and the difference in the threshold intensity for a spike to be counted. A rough comparison, neglecting the difference in solar activity, can be made if we assume an $E^{-3.5}$ differential energy spectrum to map their flux threshold for $E_{a} \geq 10 \mathrm{kev}\left(j \geq 2.5 \times 10^{7} \mathrm{el}\right.$ $\mathrm{cm}^{-2} \mathrm{sec}^{-1}$ ster $\left.^{-1}\right)$ to our energy threshold. The corresponding flux for $E_{0} \geq 425 \mathrm{kev}$ would be approximately $2.1 \times 10^{3} \mathrm{el} \mathrm{cm}^{-2} \mathrm{sec}^{-1}$ ster $^{-1}$, and the corresponding $\mathrm{D}_{1} \overline{\mathrm{D}}_{3}$ counting rate is about $140 \mathrm{sec}^{-1}$. About $15 \%$ of our spikes (average of all types) have rates larger than this value (compare Figure 9), so we should multiply the normalized occurrence scale in Figure 10 by 0.15 to make the comparison. This calculation gives about a $5 \%$ peak occurrence probability near local midnight, which compares well with the value of $\sim 4 \%$ reported by those authors. We also get qualitative agreement with their latitude and $K p$ dependences. Although the absolute numbers depend strongly on the assumed spectrum, this procedure shows that it is not unreasonable to assume that the spikes we observe at energies above $425 \mathrm{kev}$ are simply manifestations of the high-energy tails of large events observed at much lower energies.

The normalized dependence of spike occurrence on $K p$ is shown in Figure 11. Here the correction for nonuniform sampling is even more important, since there are very few passes at high $K p$. As might be expected, spikes are observed more frequently at times of high $K p$, the exception being spikes of type $2 \mathrm{~A}$, which appear to show the opposite behavior.

\section{Discussion}

The most striking feature of the data presented here is that the spikes can be grouped into several distinct populations with widely different characteristics. This grouping emphasizes the organizing power of various physical parameters, allowing more reliable and accurate descriptions of the phenomena and possible source mechanisms. We assume that there are only three possible sources for spikes observed at low altitudes: (1) local acceleration on lines of force connected to the region of observation, (2) scattering from the stably trapped population, and (3) transport from various regions of the outer magnetosphere, possibly accompanied by acceleration. These sources will be discussed in relation to each spike type.

Type 1 spikes have several characteristics that point to their most likely source: (1) by definition, they occur at latitudes below the 
trapping limit, and thus they are on closed field lines and able to exhibit trapped or pseudotrapped behavior; (2) they are not observed to persist on successive orbits, this finding indicating lifetimes less than about $100 \mathrm{~min}$; and (3) their intensities are not much larger than those of outer-zone trapped electrons. These observations are consistent with local acceleration or pitch-angle scattering from the stably trapped population into the loss cone. Although we are not able to distinguish between these alternatives on the basis of the data presented here, the second possibility appears far more attractive. Strong pitch-angle scattering is fairly well understood [Kennel and Petschek, 1966], although the details for impulsive events at the energies we observe have not been discussed explicitly. It is unlikely that the trapped $425-\mathrm{kev}$ electron flux is near the point of self-excitation of the Kennel and Petschek mechanism, but scattering at this energy might be caused by interactions with waves generated by lowerenergy particles. Local acceleration to relativistic energies would seem less likely.

Type 3 spikes present a totally different picture. Their latitude and local-time dependence strongly suggests that they are occurring near the 'last' closed field line at every local time. This suggestion then would mean that they occur on lines that approach the magnetopause or the neutral sheet, depending on the local time at the foot. The peak in occurrence just east of local noon (see Figure 10) indicates possible connection to the neutral point, where field turbulence might be expected to be large. Several experimenters (see Figure 6) have observed similar impulsive fluxes of high-energy electrons both near the magnetopause and near the neutral sheet, and Meng and Anderson [1970] have observed a sheet of electrons ( $>40 \mathrm{kev}$ ) near the magnetopause. The source mechanism(s) for these events has not been definitely established, but it seems likely that the low-altitude spikes of type 3 and the distant fluxes may be manifestations of the same process. Notice, however, that the mapping of Fairfield [1968, Figure 6] indicates that the precipitating spikes are associated not with the magnetopause but with the flanks of the magnetosphere. Both a better field model and simultaneous observations at low altitudes and near the magnetopause are needed to distinguish between these possibilities.

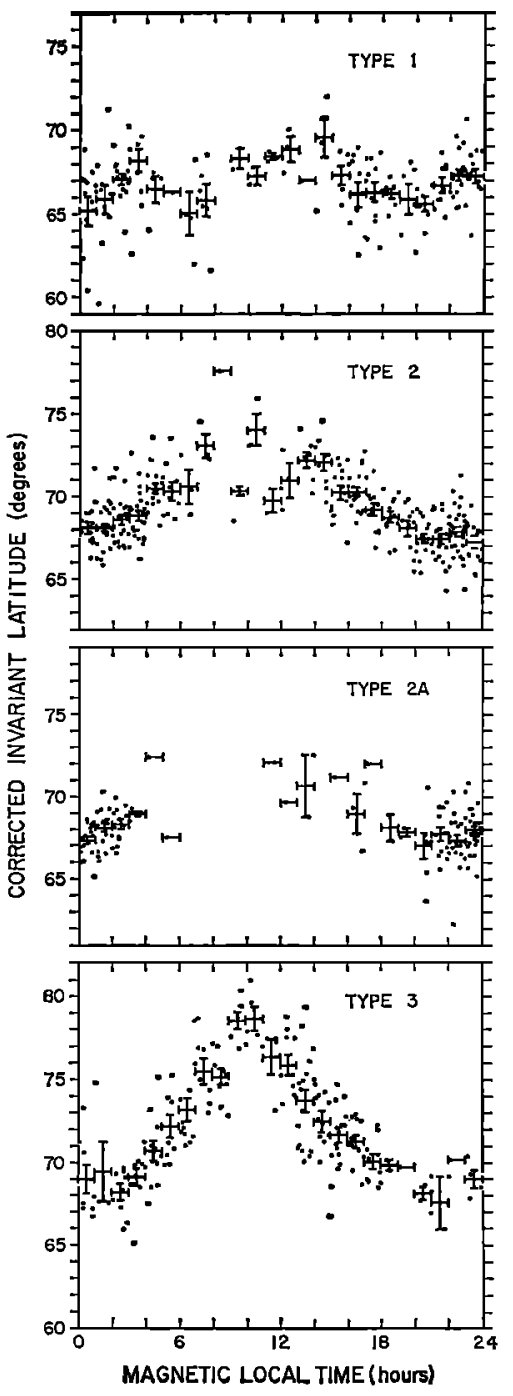

Fig. 8. Plots of 'corrected' invariant latitude ( $\Lambda$ corrected to $K p=0$ ) versus magnetic local time. Error bars have the same meaning as in Figure 7.

The type 2 spikes (and especially type 2A) present an even more interesting picture. The strong day-night asymmetry and the latitude of occurrence near midnight $\left(\Lambda \approx 67^{\circ}\right)$ indicate that these events are related to the neutral sheet and/or the cusp region. Hones et al. [1971] have observed an energetic electron spike simultaneously at $1,6.6$, and $18 R_{B}$ near local midnight. This observation confirms the interpretation that neutral-sheet and low-altitude spikes are directly related. A likely acceleration mechanism would 


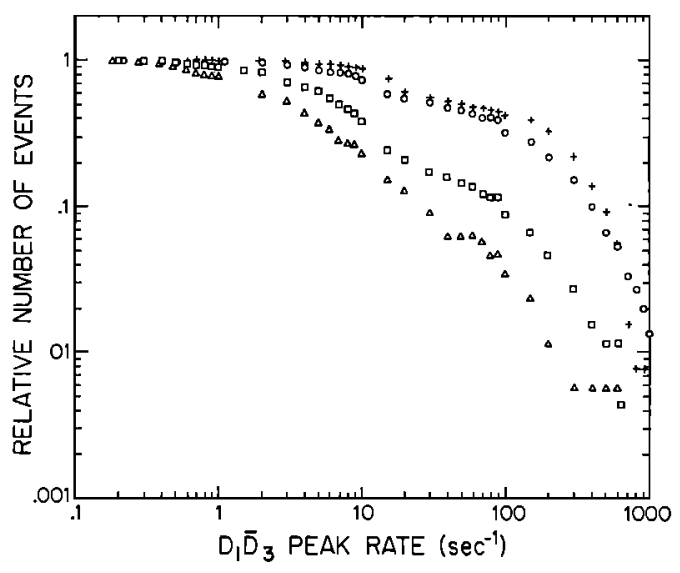

Fig. 9. Relative number of spikes larger than a given value of $D_{1} \bar{D}_{8}$. Circles, type 1 ; squares, type 2 ; plus signs, type $2 \mathrm{~A}$; triangles, type 3 .

be the merging of field lines in the neutral sheet and subsequent collapse of the stretched field lines into a dipolelike configuration, perhaps preceded by particle precipitation and field deflation at $L \approx 4-6$. This process has been suggested by Axford [1969] and observed by McPherron and Coleman [1970] and Lezniak.

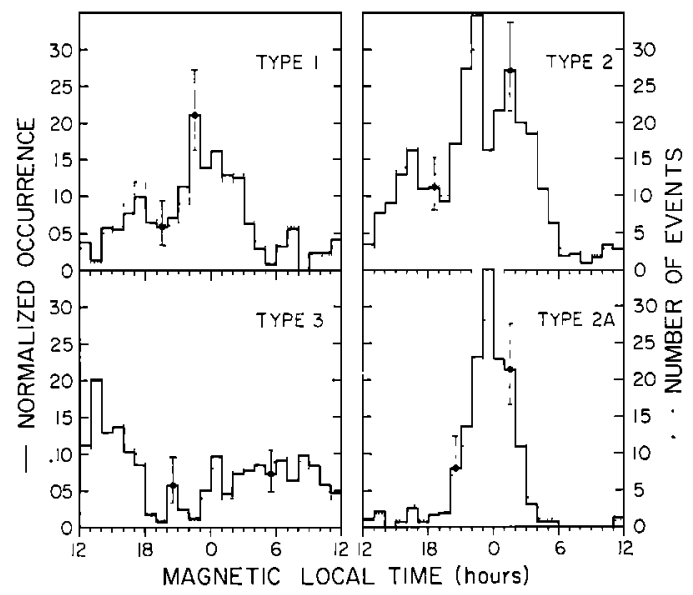

Fig. 10. Number of spikes of each types as a function of magnetic local time. Note that local midnight is in the center of the time axes, primarily to emphasize the type $2 \mathrm{~A}$ distribution. Also included are data normalized for unequal localtime coverage (see section on coverage normalization). Dotted lines, raw counts; solid lines, normalized counts. Error bars refer only to the statistics associated with the number of events observed.

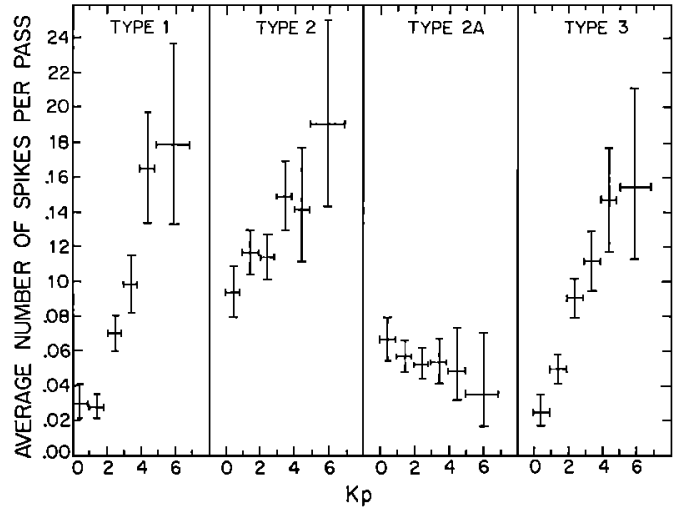

Fig. 11. Relation between spike occurrence and $K p$.

and Winckler [1970]. The results of Arnoldy and Chan [1969] seem to indicate that electron spikes observed at $6.6 R_{E}$ have drifted from an injection point at local midnight. Moreover, the observations of Hones et al. [1968] indicate that the source is probably nearer than $17 R_{E}$ to the earth. Parks [1970], however, has attributed some events of this type to strong pitch-angle diffusion.

The type $2 \mathrm{~A}$ spikes are of special interest, since their characteristically sharp cutoff on the high-latitude side and their strong tendency to occur conjugately indicate that they may define very accurately the last closed field line near local midnight. Examination of Figure 4 clearly illustrates this sharp cutoff and tendency to occur conjugately. The spike first appeared at 1910 UT during the north polar pass of revolution 827. At this time the trapping limit was at a higher latitude, but within 50-60 min it had moved down to the latitude of the spike. The configuration of the magnetosphere then apparently remained fairly stable for about 10 hours, until some time between $0500 \mathrm{UT}$ and $0720 \mathrm{UT}$, when the spike disappeared rather abruptly. This occurrence should not be assumed to be a common one, however, since only two events of such long duration were observed and since the scarcity of type 2 events on the dayside and the short lifetimes of type 1 and type 3 spikes indicate that stable trapping of spike particles is uncommon. The results of Arnoldy and Chan [1969] indicate that the decay time at $6.6 R_{\bar{E}}$ is probably about the same as the drift period for electron energies of $50 \leq E_{e} \leq 150 \mathrm{kev}$. 


\section{Summary}

The vertical particle telescope on Ogo 4 has detected numerous spikes of precipitating electrons with energies above $425 \mathrm{kev}$. These spikes occur within a few degrees of the high-latitude boundary of the outer zone and have characteristic widths on the order of $1^{\circ}$ in latitude. The time scale for buildup and decay of an event is of the order of several minutes to several hours.

It is instructive to group the spikes into several populations on the basis of their latitude with respect to the outer-zone boundary. The local-time dependence is highly nonuniform, at least 5 times as many events occurring near 2200 MLT as near 1000 MLT. The disparity is much greater (about 12:1) if only those spikes occurring at or below the outer-zone boundary are considered.

The average invariant latitude of occurrence is $67^{\circ}-68^{\circ}$, but it is strongly dominated by the large number of spikes occurring near local midnight. They tend to occur at higher latitudes and to have a larger spread in latitudes near local noon. The effect of local time on latitude is strongest for the spikes that occur above the trapping boundary.

Latitude of occurrence is also affected by geomagnetic disturbance, as indicated by the $K p$ index. The latitude tends to be decreased by about $0.6^{\circ} \pm 0.3^{\circ}$ per unit $K p$, and this effect is stronger near local noon than near midnight.

Fits to assumed power-law spectra indicate an average spectral index of 4 to 5 (or $e$-folding energies of 130 to $170 \mathrm{kev}$ for exponential spectra), except for special large characteristically shaped events (type 2A) that occur at the trapping boundary near local midnight and have an index around 3 for power-law spectra (or 260 -kev $e$-folding energy for exponential). The median flux $\left(E_{\mathrm{e}}>425 \mathrm{kev}\right)$ is about $150 \mathrm{el} \mathrm{\textrm {cm } ^ { - 2 }}$ $\mathrm{sec}^{-1}$ ster $^{-1}$, except for the low-latitude (type 1) events and the special (type 2A) events mentioned above, which are about 5 times as large.

Acknowledgments. We gratefully acknowledge the collaboration and support of Drs. J. A. Simpson and C. Y. Fan during various stages of the joint University of Chicago/California Institute of Technology program. The instrument was constructed by the Laboratory of Astrophysics and Space Research of the University of Chicago.

This work was performed under contract NAS53095 and grants NGL 05-002-007 and NGR 05-
002-160. J. W. Brown received valuable support from the National Science Foundation.

The Editor thanks K. A. Anderson and L. A. Frank for their assistance in evaluating this paper.

\section{REFERENCES}

Anderson, H. R., P. D. Hudson, and J. E. McCoy, Observations of Pogo ion chamber experiment in the outer radiation zone, J. Geophys. Res., $73,6285,1968$.

Anderson, K. A., Energetic electron fluxes in the tail of the geomagnetic field, J. Geophys. Res., $70,4741,1965$.

Anderson, K. A., H. K. Harris, and R. J. Paoli, Energetic electron fluxes in and beyond the earth's outer magnetosphere, J. Geophys. Res., $70,1039,1965$.

Arnoldy, R. L., and K. W. Chan, Particle substorms observed at the geostationary orbit, $J$. Geophys. Res., 74, 5019, 1969.

Axford, W. I., Magnetospheric convection, Rev. Geophys. Space Phys., \%, 421, 1969.

Evans, L. C., J. L. Fanselow, and E. C. Stone, User's notes for experiments C-08 and D-08 rate plots, Intern. Rep. 11, Space Radiat. Lab., Calif. Inst. of Technol., Pasadena, 1970.

Fairfield, D. H., Average magnetic-field configuration of the outer magnetosphere, J. Geophys. Res., 73, 7329, 1968.

Fan, C. Y., G. Gloeckler, and J. A. Simpson, Acceleration of electrons near the earth's bow shock and beyond, J. Geophys. Res., 71, 1837, 1966.

Frank, L. A., On the local-time dependence of outer radiation zone electron $(E>1.6 \mathrm{Mev})$ intensities near the magnetic equator, J. Geophys. Res., 70, 4131, 1965.

Frank, L. A., and J. A. Van Allen, Measurements of energetic electrons in the vicinity of the sunward magnetospheric boundary with Explorer 14, J. Geophys. Res., 69, 4923, 1964.

Fritz, T. A., and D. A. Gurnett, Diurnal and latitudinal effects observed for $10-\mathrm{kev}$ electrons at low satellite altitudes, J. Geophys. Res., 70, $2485,1965$.

Hoffman, R. A., Low-energy electron precipitation at high latitudes, J. Geophys. Res., 74, 2425, 1969.

Hoffman, R. A., and D. S. Evans, Field-aligned electron bursts at high latitudes observed by Ogo 4, J. Geophys. Res., 73, 6201, 1968.

Hones, E. W., Jr., S. Singer, and C. S. R. Rao, Simultaneous observations of electrons $(E>$ $45 \mathrm{kev}$ ) at 2000-kilometer altitude and at 100,000 kilometers in the magnetotail, J. Geophys. Res., 78, 7339, 1968.

Hones, E. W., Jr., R. H. Karas, L. J. Lanzerotti, and S.-I. Akasofu, Magnetospheric substorms on September 14, 1968, J. Geophys. Res., 76, $6765,1971$.

Jensen, D. C., and J. C. Cain, An interim geomag- 
netic field (abstract), J. Geophys. Res., 67, 3568, 1962.

Kennel, C. F., and H. E. Petschek, Limit on stably trapped particle fluxes, J. Geophys. Res., 71, 1, 1966.

Lezniak, T. W., and J. R. Winckler, Experimental study of magnetospheric motions and the acceleration of energetic electrons during substorms, J. Geophys. Res., 75, 7075, 1970.

Lupton, J. E., and E. C. Stone, Electron scattering effects in typical cosmic ray telescopes, $I E E E$ Trans. Nucl. Sci., NS-19, 562, 1972.

McCoy, J. E., High-latitude ionization spikes observed by the Pogo ion chamber experiment, J. Geophys. Res., 74, 2309, 1969.

McDiarmid, I. B., and J. R. Burrows, Electron fluxes at 1000 kilometers associated with the tail of the magnetosphere, J. Geophys. Res., 70, 3031, 1965.

McDiarmid, I. B., and J. R. Burrows, Local time asymmetries in the high-latitude boundary of the outer radiation zone for the different electron energies, Can. J. Phys., 46, 49, 1968.

McPherron, R. L., and P. J. Coleman, Inward collapse of the magnetic tail during magnetospheric substorms (abstract), Eos Trans. AGU, $51,402,1970$.
Meng, C.-I., and K. A. Anderson, A layer of energetic electrons ( $>40 \mathrm{kev}$ ) near the magnetopause, J. Geophys. Res., 75, 1827, 1970.

Montgomery, M. D., S. Singer, J. P. Conner, and E. E. Stogsdill, Spatial distribution, energy spectra, and time variations of energetic electrons ( $E>50 \mathrm{kev}$ ) at 17.7 earth radii, Phys. Rev. Lett., 14, 209, 1965.

Murayama, T., and J. A. Simpson, Electrons within the neutral sheet of the magnetospheric tail, J. Geophys. Res., 78, 891, 1968.

Parks, G. K., The acceleration and precipitation of Van Allen outer-zone energetic electrons, $J$. Geophys. Res., 75, 3802, 1970.

Retzler, J., and J. A. Simpson, Relativistic electrons confined within the neutral sheet of the geomagnetic tail, J. Geophys. Res., 74, 2149, 1969.

Rosen, A., The radiation-belt boundary near solarcycle maximum as determined from the trapping of energetic electrons, J. Geophys. Res., 70, 4793, 1965.

(Received January 20, 1972; accepted March 15, 1972.) 